\title{
APPROXIMATE DECOUPLING OF A CONDITION FOR VOLTAGE COLLAPSE
}

Ian Dobson

Department of Electrical and Computer Engineering

1415 Johnson Drive

University of Wisconsin-Madison

Madison, WI 53706

Email: dobson@ie.engr.wisc.edu

\begin{abstract}
An index for voltage collapse in a power system model with a general dynamic load is roughly approximated and simplified using the standard $P-\delta, Q-V$ decoupling. The simplified index measures the closeness to bifurcation of the load reactive power balance equations.
\end{abstract}

\section{INTRODUCTION}

Voltage collapse in an electric power system is the progressive decrease in voltage magnitudes at load buses leading to blackout. For reviews and references see $[1,2,3]$. Although the specific mechanisms of voltage collapse are not clear, voltage collapse is associated with reactive power supply problems [4] and bifurcation of equations representing the power system $[1,5]$. For example, in a power system model in the form of differential equations, voltage collapse is associated with bifurcation of equilibria $[6,7]$. The closeness to bifurcation can be measured by the determinant $\Delta$ of the system Jacobian and the condition for bifurcation and a subsequent voltage collapse is $\Delta=0$.

In this short paper we assume a form for the power system differential equations and calculate the index $\Delta$. We then make approximations to reduce the bifurcation condition $\Delta=0$ to the bifurcation of the load reactive power balance equations. This shows the relation between the voltage collapse bifurcation condition for a power system modelled as differential equations and modelled by static power balance equations. Thus we reduce the voltage collapse index $\Delta$ to a simpler, approximate index measuring the closeness to bifurcation of the load reactive power balance equations. The approximation uses the $P-\delta, Q-V$ decoupling commonly used in transient stability and load flow studies.

The main purpose in deriving a simpler, approximate index for voltage collapse is to study the qualitative effect of the various subsystems or parameters on the closeness to voltage collapse. (Before the simplification, the index is too complicated for this study.) For example, the effect on the simplified index of generators reaching their reactive power limits could be studied. Moreover the approximations made when deriving the index have the effect of eliminating variables in the power system model which are of only secondary importance in causing voltage collapse.

\section{POWER SYSTEM MODEL}

We suppose that there are $m+1$ generator buses numbered $0,1,2, \ldots, m$ with bus 0 a slack bus and $n$ load buses numbered $1,2 \ldots, n$. The generator voltage angles and magnitudes are written as $\delta_{G}=\left(\delta_{G 1}, \ldots, \delta_{G m}\right)^{T}$ and $V_{G}=\left(V_{G 1}, \ldots, V_{G m}\right)^{T}$ and the load voltage angles and magnitudes are written as $\delta_{L}=$ $\left(\delta_{L 1}, \ldots, \delta_{L n}\right)^{T}$ and $V_{L}=\left(V_{L 1}, \ldots, V_{L n}\right)^{T}$. We also write $\delta=$ $\left(0, \delta_{G}^{T}, \delta_{L}^{T}\right)^{T}=\left(\delta_{0}, \delta_{1}, \ldots, \delta_{m+n}\right)^{T}$ and $V=\left(V_{0}, V_{G}^{T}, V_{L}^{T}\right)^{T}=$ $\left(V_{0}, V_{1}, \ldots, V_{m+n}\right)^{T}$ and $B_{i j} \angle \theta-\frac{\pi}{2}$ for the admittance of the line joining $i$ and $j$.

The generators are modelled by the swing equations

$$
\begin{aligned}
\dot{\delta}_{G} & =\omega \\
M \dot{\omega} & =-D \omega+P_{M}-P_{G}(\delta, V)
\end{aligned}
$$

where $M=\operatorname{diag}\left\{M_{1}, \ldots, M_{m}\right\}, D=\operatorname{diag}\left\{D_{1}, \ldots, D_{m}\right\}$ and $P_{M}=\left(P_{M 1}, \ldots, P_{M m}\right)^{T}$ are the generator inertias, dampings and mechanical powers respectively. $P_{G}=\left(P_{G 1}, \ldots, P_{G m}\right)^{T}$ is the electrical power supplied to the network where

$$
P_{G j}=\sum_{i=0}^{m+n} B_{i j} V_{i} V_{j} \sin \left(\delta_{i}-\delta_{j}+\theta_{i j}\right), \quad j=1, \ldots, m
$$

Write $P_{L k}$ and $Q_{L k}$ for the real and reactive powers supplied by the network to load bus number $k, k=1, \ldots, n$. Then

$$
\begin{aligned}
& P_{L k}=-\sum_{i=0}^{m+n} B_{i m+k} V_{i} V_{L k} \sin \left(\delta_{i}-\delta_{L k}+\theta_{i m+k}\right) \\
& Q_{L k}=-\sum_{i=0}^{m+n} B_{i m+k} V_{i} V_{L k} \cos \left(\delta_{i}-\delta_{L k}+\theta_{i m+k}\right)
\end{aligned}
$$

Each load bus is modelled by the general form

$$
\left(\begin{array}{c}
P_{L k} \\
Q_{L k}
\end{array}\right)=E_{k}\left(\begin{array}{c}
\dot{\delta}_{L k} \\
\dot{V}_{L k}
\end{array}\right)+\left(\begin{array}{c}
f_{k}\left(V_{L k}\right) \\
g_{k}\left(V_{L k}\right)
\end{array}\right)
$$

where $E_{1}, \ldots, E_{n}$ are invertible $2 \times 2$ constant matrices. We write $P_{L}=\left(P_{L_{1}}, \ldots, P_{L n}\right)^{T}$ and $Q_{L}=\left(Q_{L 1}, \ldots, Q_{L n}\right)^{T}$ to put (2.2) in vector form:

$$
\left(\begin{array}{c}
P_{L} \\
Q_{L}
\end{array}\right)=E\left(\begin{array}{l}
\dot{\delta}_{L} \\
\dot{V}_{L}
\end{array}\right)+\left(\begin{array}{l}
f\left(V_{L}\right) \\
g\left(V_{L}\right)
\end{array}\right)
$$

where $E$ is the matrix $\operatorname{diag}\left\{E_{1}, \ldots, E_{n}\right\}$ with its rows and columns suitably permuted. $E$ is invertible since $E_{1}, \ldots, E_{n}$ are invertible. Equation (2.3) is a dynamic load model which generalises the induction motor model of Walve [8] used in $[6,7]$. It is necessary to assume a general form for a dynamic load model because little seems to be known about such models.

Note that the (static) load reactive power balance equations $0=Q_{L}(V, \delta)-g\left(V_{L}\right)$ are obtained from (2.3) by setting $\dot{\delta}=\dot{V}_{L}=0$. For $\delta$ and $\theta$ small and of the same order, $Q_{L k}$ may be approximated to $O\left(\delta^{2}\right)$ by $\tilde{Q}_{L k}=-\sum_{i=0}^{m+n} B_{i m+k} V_{i} V_{L k}$. The approximate load reactive power balance equations are then

$$
0=\tilde{Q}_{L}(V)-g\left(V_{L}\right)
$$

\section{APPROXIMATE DECOUPLING}

The power system differential equations may be obtained by rearranging equations (2.1) and (2.3).

$$
\begin{aligned}
\dot{\delta}_{G} & =\omega \\
\dot{\omega} & =-M^{-1} D \omega+M^{-1} P_{M}-M^{-1} P_{G}(\delta, V) \\
\left(\begin{array}{c}
\dot{\delta}_{L} \\
\dot{V}_{L}
\end{array}\right) & =E^{-1}\left(\begin{array}{c}
P_{L}(\delta, V)-f\left(V_{L}\right) \\
Q_{L}(\delta, V)-g\left(V_{L}\right)
\end{array}\right)
\end{aligned}
$$


The determinant $\Delta$ of the Jacobian $J$ of (3.1) simplifies to

$$
\Delta=(-1)^{m+1}\left|\begin{array}{cc}
M^{-1} & 0 \\
0 & E^{-1}
\end{array}\right|\left|\begin{array}{cccc}
\frac{\partial P_{G}}{\partial \delta_{G}} & \frac{\partial P_{G}}{\partial \delta_{L}} & \vdots & \frac{\partial P_{G}}{\partial V_{L}} \\
\frac{\partial P_{L}}{\partial \delta_{G}} & \frac{\partial P_{L}}{\partial \delta_{L}} & \vdots & \frac{\partial P_{L}}{\partial V_{L}}-\frac{\partial f}{\partial V_{L}} \\
\cdots & \cdots & \cdot & \cdots \\
\frac{\partial Q_{L}}{\partial \delta_{G}} & \frac{\partial Q_{L}}{\partial \delta_{L}} & \vdots & \frac{\partial Q_{L}}{\partial V_{L}}-\frac{\partial g}{\partial V_{L}}
\end{array}\right|
$$

Write $k=(-1)^{m+1}|M|^{-1}|E|^{-1}$ and $\Delta=k\left|\begin{array}{cc}A & B \\ C & D\end{array}\right|$

where $A, B, C, D$ are the submatrices indicated in (3.2).

Now we assume that $\delta$ and $\theta$ are small and of the same order. Then inspection of $\frac{\partial P_{G}}{\partial V_{L}}, \frac{\partial P_{L}}{\partial V_{L}}, \frac{\partial Q_{L}}{\partial \delta_{G}}, \frac{\partial Q_{L}}{\partial \delta_{L}}$ shows that each of these matrices is $O(\delta)$. If we further assume that $\frac{\partial f}{\partial V_{L}}$ is $O(\delta)$, then each element of matrices $B$ and $C$ is $O(\delta)$. Now we claim that $\Delta=k|A||D|+O\left(\delta^{2}\right)$. (This may be proved by Laplace expanding [9] the determinant in (3.3) as the sum of products of $(m+n) \times(m+n)$ minors from the first $m+n$ columns and their complementary minors from the last $n$ columns and noting that the minor and complementary minors in each product contribute a term from each of $B$ and $C$ except for the case of the product $|A||D|$.) The matrix $D$ is given by $D=\frac{\partial Q_{L}}{\partial V_{L}}-\frac{\partial g}{\partial V_{L}}$ and can be approximated to $O\left(\delta^{2}\right)$ by $\tilde{D}=\frac{\partial \tilde{Q}_{L}}{\partial V_{L}}-\frac{\partial g}{\partial V_{L}}$ so that

$$
\Delta=k|A||\tilde{D}|+O\left(\delta^{2}\right)
$$

Hence an approximate condition for bifurcation is $|A|=0$ or $|\tilde{D}|=0$. However we do not expect $|A|=0$ in a voltage collapse. (One argument for this is that $|A|=0$ implies the existence of an "O( $\delta)$ eigenvector" $x=\left(x_{G}, 0, x_{L}, 0\right)^{T}$ of the Jacobian $J$ which satisfies $J x=O(\delta)$. Since $x$ points in an angle direction, such a bifurcation would be associated with pole slip rather than voltage collapse (see [6]).) Thus $|\tilde{D}|=0$ is an approximate condition for voltage collapse. The voltage collapse index $|\tilde{D}|$ depends only on voltage magnitudes $V_{L}$ and is 'decoupled' from the voltage angles. $\left(|D|\right.$ also depends on $V_{G}$ but these are constant in power system model (3.1).) Moreover, $|\tilde{D}|=0$ is the condition for bifurcation of the approximate load reactive power balance equations (2.4).

\section{DISCUSSION AND CONCLUSIONS}

The main approximations used in Section 3 are of small voltage angle differences across lines and that $\frac{\partial f}{\partial V_{L}}$ is $O(\delta)$. The small line angle assumption is a good assumption for normal operating conditions; it is less good for the study of voltage collapse because voltage collapse typically occurs when the system is heavily loaded. The assumption that $\frac{\partial f}{\partial V_{L}}$ is $O(\delta)$ is the as sumption that the real power demand of the load is relatively insensitive to load voltage magnitude. This may be an appropriate simplifying approximation and several authors have used it $[10,11]$. However a load modelling study [12] indicates values of $\frac{\partial f}{\partial V_{L}}$ that are not negligible, typically about half of $\frac{\partial g}{\partial V_{L}}$ for a composite load. If $\frac{\partial f}{\partial V_{L}}$ is $O(1)$, then the calculation of Section 3 proceeds as before but is only accurate to $O(\delta)$. We conclude that the approximations seem crude but appropriate for qualitative studies of voltage collapse.

This short paper shows how a voltage collapse index for a power system modelled as differential equations may be reduced to a simpler approximate index describing the bifurcation of the load reactive power balance equations. The approximate index is expected to be useful in qualitative studies of the main causes of voltage collapse and in calculating approximate solutions for bifurcation points in voltage collapse studies. A simple example of the latter application is in [7]. Of course, the approximate index is also easier to compute and is a candidate for monitoring a power system for closeness to voltage collapse. However, it is not clear that the approximate index is accurate enough for this purpose.

This paper uses a general dynamic load model but either omits or models very simply other subsystems that may also be important causes of voltage collapse. For example, tap-changer dynamics and generator limits are not modelled. Future work will attempt to simplify voltage collapse indices for power system models taking account of all the subsystems thought to cause voltage collapse. If the reduction to an approximate index describing the bifurcation of the load reactive power balance equations is still possible, then this would demonstrate analytically the importance of load reactive power balance in voltage collapse.

\section{ACKNOWLEDGEMENTS}

The author gratefully acknowledges support in part from NSF under grant number ECS-8352211 and the Graduate School at the University of Wisconsin-Madison.

\section{REFERENCES}

[1] Kwatny, H.G., Pasrija, A.K., Bahar, L.Y., "Static bifurcations in electric power networks: loss of steady-state stability and voltage collapse," IEEE Transactions on Circuits and Systems, vol. CAS-33, no. 10, October 1986, pp. 981-991.

[2] Chiang, H.D., Wu, F.F., "On voltage stability," Proc. 1986 IEEE ISCAS, vol. 3, May 1986, pp. 1339-1343.

[3] Mercede, F., Chow, J.C., Yan, H., Fischl, R., "A framework to predict voltage collapse in power systems," IEEE PEB Winter Power Meeting, New York, NY, 1988.

[4] Lachs, W.R., "Insecure system reactive power balance analysis and countermeasures", IEEE Transactions on Power Apparatus and Systems, vol. PAS-104, no. 9, Sept 1985, pp. 2413-2419.

[5] Tamura, Y.,Mori, H., Iwamoto, S., "Relationship between voltage instability and multiple load flow solutions in electric power systems," IEEE Transactions on Power Apparatus and Systems, vol. PAS-97, no. 6, May 1983, pp. 1115-1125.

[6] Dobson, I., Chiang, H.-D., "Towards a Theory of Voltage Collapse in Electric Power Systems," to appear in System and Control Letters, 1990.

[7] Dobson, I., Chiang, H.-D., Thorp, J.S., Fekih-Ahmed, L., "A Model of Voltage Collapse in Electric Power Systems," Pro. ceedings of the 27th IEEE Control and Decision Theory Conference, Austin, Texas, Dec. 1989, pp. 2104-2109.

[8] Walve, K., "Modelling of Power System Components at Severe Disturbances," CIGRÉ paper 38-18, International Conference on Large High Voltage Electric Systems, August 1986.

[9] Maclane, S., Birkhoff, G., Algebra, Chelsea, N.Y., 1988.

[10] Concordia, C., Ihara, S., "Load Representation in Power System Stability Studies", IEEE Transactions on Power Apparatus and Systems, vol. PAS-101, no. 4, April 1982, pp. 969-977.

[11] DeMarco, C.L., Bergen, A.R., "A security measure for random load disturbances in nonlinear power system models," IEEE Transactions on Circuits and Systems, vol. CAS-34, no. 12, December 1987, pp. 1546-1557.

[12] Begovic, M.M., Phadke, A.G., "Dynamic Simulation of Voltage Collapse," Proc. PICA, May 1989. 